\title{
How do red and infrared low-level lasers affect folliculogenesis cycle in rat's ovary tissue in comparison with clomiphene under in vivo condition
}

\author{
Paria Naseri $^{1,2}$ - Alireza Alihemmati ${ }^{2,3}$ - Seyed Hossein Rasta ${ }^{1,2,4}$
}

Received: 27 May 2017 / Accepted: 24 July 2017 /Published online: 11 August 2017

(C) The Author(s) 2017. This article is an open access publication

\begin{abstract}
Folliculogenesis is a cycle that produces the majority of oocyte. Any disruption to this cycle leads to ovulation diseases, like polycystic ovarian syndrome (PCOS). Treatments include drugs and surgery; lasers have also been used complementarily. Meanwhile, still there is no definite treatment for PCOS. This study investigated the photo-bio stimulation effect of near-infrared and red low-level laser on producing follicles and compared the result with result of using common drug, clomiphene. Therefore, the aim of this study was to propose the use of lasers autonomously treatment. So, there was one question: how do lasers affect folliculogenesis cycle in rat's ovary tissue? In this study, 28 rats were assigned to four groups as follows: control (CT), clomiphene drug (D), red laser (RL), and near-infrared laser (NIRL). Afterwards, 14 rats of RL and NIRL groups received
\end{abstract}

Seyed Hossein Rasta

s.h.rasta@abdn.ac.uk

Paria Naseri

mph.phd16@gmail.com

Alireza Alihemmati

hemmatti@yahoo.com

1 Department of Medical Physics and Department of Medical Bioengineering, Tabriz University of Medical Sciences, Tabriz, Iran

2 Stem Cell Research Centre, Tabriz University of Medical Sciences, Tabriz, Iran

3 Department of Anatomical Sciences, Histology and Embryology, Faculty of Medicine, Tabriz University of Medical Sciences, Tabriz 51666, Iran

4 School of Medical Sciences, University of Aberdeen, Aberdeen, UK laser on the first 2 days of estrous cycle, each 6 days, for 48 days. During treatment period, each rat received energy density of $5 \mathrm{~J} / \mathrm{cm}^{2}$. Seven rats in D group received clomiphene. After the experiment, lasers' effects at two wavelengths of 630 and $810 \mathrm{~nm}$ groups have been investigated and compared with clomiphene and $\mathrm{CT}$ groups. Producing different follicles to complement folliculogenesis cycle increased in NIRL and RL groups, but this increase was significant only in the NIRL group. This indicates that NIRL increases ovarian activity to produce oocyte that certainly can be used in future studies for finding a cure to ovarian negligence to produce more oocyte and treat diseases caused by it like PCOS.

Keywords Near-infrared laser · Red laser · Clomiphene drug · Folliculogenesis cycle $\cdot$ Polycystic ovarian syndrome $\cdot$ Rat ovary tissue

\section{Introduction}

Folliculogenesis is a cycle in which primordial follicles grow and produce graffian follicles in several stages and eventually produce the majority of oocyte [1]. Primordial follicles are embryonic [2] since there were a million of them resting follicle in every woman's body at birth [3]. So, before puberty, mammalian ovary contains these follicles [4]. There are a lot of non-growing primordial follicles in their ovary (oocytes surrounded by flattened pre-granulosa cells) [5] most of which remain until puberty; then, they may either degenerate or activate and grow towards primary, secondary, tertiary, and quaternary stages (with an antral cavity) [6]. Following activation, primordial follicles develop and give rise to primary follicles (i) and then secondary follicles (ii). Next, by the stimulation of 
gonadotropin (FSH and luteinizing hormone (LH)), multilayered antral follicles containing antral cavities are produced (iii); in the last stage, granulosa cells are demarcated and cause a single large antral cavity to be formed and the oocyte, ready for ovulation, reaches its final growth in the graffian follicle (iv); in other words, folliculogenesis cycle is completed [1, 7, 8]. Disorder in folliculogenesis cycle causes several diseases; polycystic ovarian syndrome (PCOS) is an important one [9]. In the reproductive age, 5 to $10 \%$ of reproductive age woman with PCOS are infertile $[10,11]$. Search for a definite treatment of PCOS started in the early 1930s and still continues $[11,12]$. The available modalities for induction of ovulation are weight reduction, clomiphene citrate, gonadotropin therapy, insulin sensitizer as metformin, and finally, ovarian drilling using laser or bipolar diathermy. Despite these treatment modalities, there are still cases resistant to treatment; this raises the need for a new treatment method [9, 13-15].

Different lasers can be used for PCOS drilling including argon, KTP, ND:YAG, and $\mathrm{CO}_{2}$. All of these stimulate ovary to increase activation of folliculogenesis cycle to ovulation. Lasers are also used to treat ovarian malignant and cancer [16, 17-19]. Degrees of response are variable [9].

Lasers have already been used as complement therapies, applied with medication or surgery, but they are also effective for ovarian stimulation $[11,16,17,20]$. The direct effect of lasers, alone, on folliculogenesis cycle has not been investigated. So, the purpose of this study is to investigate the effect of lasers without surgery or drug on folliculogenesis cycle.

\section{Method and materials}

\section{Animals}

Initially, 28 female Wistar rats which have reached sexual maturity, with the average age of 9 weeks and in weight range of 150-300 g, were obtained from animal's house of Tabriz University of Medical sciences (TUMS), Medical Physics Department. Then, rats' general health was examined by estrous test in order to be in the same phase of menstrual cycle. They were randomly assigned to four groups $(n=7)$ : control group (CT), without any intervention; clomiphene drug group (D), stimulated by clomiphene; red laser group (RL), stimulated by visible laser in red spectrum; and nearinfrared laser (NIRL), stimulated by laser in the nearinfrared spectrum. Afterwards, they were taken care of for 1 week on environmental conditions with free access to food and water; temperature $\left(25-27^{\circ} \mathrm{C}\right)$ and humidity were kept steady so that the rats would adapt to conditions of the new place. After a week, rats were weighed to ensure they remain in the range. Their behavior in the cage was examined during the experiment. The research protocol of this study was approved by Ethics in Research Committee of Tabriz University of Medical Sciences TUMS, under code number: TBZMED.REC.1394.238.

\section{Preparation process}

Rats were painted for each group and labeled from one to seven. Then, their hairs in RL and NIRL groups were shaved and then the shaved areas were washed using Betadine. The point for laser radiation on their skin was determined and marked by marker. During the experiment, rats were in their own cages, expecting intervention or box cleaning (three times per week). Intervention on rats was done at the beginning of menstrual cycle. The preparation method in this study was designed based on references [9, 11, 20-25].

\section{Laser treatment}

Fourteen rats of RL and NIRL groups received laser on the first 2 days of estrous cycle on specified times (each 6 days, for 48 days). Before doing the experiment, dose transfer percent and irradiation time were determined by a primary experiment. The probes were done by two diode lasers (MUSTANG 2000 +, Moscow, Russia), 630 and $810 \mathrm{~nm}$, by $33 \pm 2$ and $212 \pm 2$ power output with $10 \mathrm{~mm}$ diameter of nuzzle for both probes, the laser beam area was $0.79 \mathrm{~cm}^{2}$, and power densities were $41.77 \mathrm{mw} / \mathrm{cm}^{2}$ for red laser and $268.35 \mathrm{mw} / \mathrm{cm}^{2}$ for NIR laser beams. Continuous pulse laser mode has been found to be optimal for increasing the activity of protein, ATP synthesis, and cell growth [21, 23]. Irradiation was done for 142.2 and $28 \mathrm{~s}$ for RL and NIRL groups, respectively. The laser probes were placed directly on the marked part of the skin with perpendicular angle (Fig. 1a). Henceforth, the same dose of laser was transferred on ovary for two groups; however, the percentage of transferred dose and irradiation time was already determined by a primary experiment. Water was used to provide coupling media for matching impedance between the laser probe and skin of rats. During the treatment, they were fixed in a Plexiglas restrainer designed for this study (Fig. 1b). The average dose reaching the target was $5 \mathrm{~J} / \mathrm{cm}^{2}$ that was found to be optimal for increasing the activity of protein, ATP synthesis, and cell growth [21]. In this study, we introduced the irradiated time for two groups, red and infrared by calculating average dose to be sure how much the average dose has been reached the target. Transcranial low-level laser therapy (LLLT) treatment was done 16 times in 8 weeks. Also, the marked skin area thickness was measured to calculate skin attenuation coefficient by Lambert-Beer equation.

$I=I_{0} e^{-\mu x}$

$\mu x=\ln \frac{I_{0}}{I}$ 
Fig. 1 a Laser irradiation: This image shows style of laser irradiation during the experiment. b Rat restrainer: this restrainer designed for this study. It is approved by Ethics and Research Committee of TUMS (code number:

TBZMED.REC.1394.238)
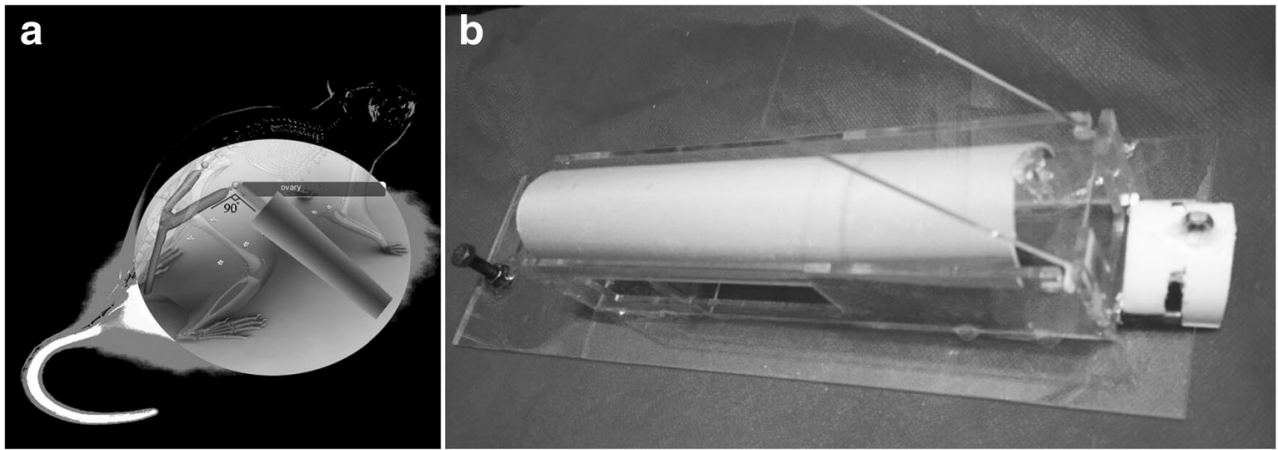

\section{Clomiphene treatment}

Seven rats in D group received clomiphene drug. This drug can stimulate folliculogenesis cycle and increase sex hormone activation and consequently increase fertility [26]. The applied dose was $1 \mu \mathrm{g} / \mathrm{kg}$ in $1 \mathrm{~cm}^{3}$ water solution. They received drug when RL and NIRL groups were undergoing irradiation. It was determined based on previously published protocol about clomiphene application [26].

\section{Treatment process}

Except for the treatments, all conditions were similar for all rats.

After the experiment, rats were weighted again. After an overnight food deprivation, each animal was anesthetized with an intraperitoneal injection of ketamine/xylazine (60/ $10 \mathrm{mg} / \mathrm{kg}$ ). Its blood serum was derived, and the ovary was immediately removed and rinsed in a cold saline and weighed. The ovary tissue was divided into two parts: one parts was frozen by liquid nitrogen and stored in $-80{ }^{\circ} \mathrm{C}$ refrigerator until the biochemical assay and the other part was immersed in $10 \%$ formalin, dehydrated in ethanol, cleared in xylene, and embedded in paraffin for histopathological evaluations. After tissue processing steps, several serial sections of ovary $(5 \mu \mathrm{m}$ thicknesses) were prepared and stained by hematoxylin and eosin (H\&E) for microscopic observations and studies. The thickness of media tunica was measured using Motic Images version 2.0 and light microscope. The stained sections were anonymously evaluated by a histologist, see also [27].

The samples were evaluated and analyzed with the statistical, SPSS software. The significance of the data was determined and table and chart were drawn, accordingly.

\section{Blood serum measurement}

Blood samples were taken by cardiac puncher and centrifuged (AZMA Co., Iran-Pars) at $3600 \mathrm{rpm}$ at room temperature for
$10 \mathrm{~min}$. Then, they were stored at $-80^{\circ} \mathrm{C}$ until the assay. They were transferred to veterinary clinic (Dr MUSAVI CLINICE, TABRIZ, IRAN, Dr. Eshrat Khah et al.) for sex hormone measurements. The kit (LH ELISA KIT, FSH ELISA KIT, E2 AccuBind ELISA, and Progesterone AccuBind ELISAPishtaz Teb-Iran) is used for counting the amount of hormones, LH, FSH, estrogen, and progesterone, which influence folliculogenesis cycle to ovulation [28-33].

\section{Laser beam transmission measurement}

Ovary and skin samples were taken and data were analyzed by a statistical program. Also, attenuation coefficient was calculated by Lambert-Beer law. By using that, laser absorption has been carried out to a percentage reaching potential that ovaries were calculated. Each data was obtained by three repetitions. They were compared with the standard information. Skin thickness was measured by a standard caliper (Pittsburgh 6in. digital caliper with $0.03 \mathrm{~mm}$ precision).

\section{Statistical analysis}

The number of different follicles and blood hormone levels, LH, FSH, estrogen, and progesterone was statistically analyzed by the IBM SPSS statistics 19 software.

Once, Tukey's HSD test and, another time, analysis of variance (ANOVA Post Hot) were used. All data were reported as mean $\pm \mathrm{SD}$. Significance level was set at $P<0.05$.

\section{Result}

For histological investigations, changes in the primary, multilaminar, secondary, and graffian follicles were studied and for hormonal investigations, follicle stimulation hormones, luteinizing, estrogen, and progesterone hormones were taken into consideration. 


\section{Histological investigations}

\section{Primary follicle}

The number of PF increased significantly in RL and NIRL groups, compared with CT and D groups. This increase was equal in the RL and NIRL groups (Fig. 2a), and hence, the difference between these two groups was not significant. Also, there was an increase in D group but this growth was less than RL and NIRL groups and did not differ significantly from the CT (Table 1). As can be inferred from the table, increase in the follicle of RL and NIRL groups was approximately 2.02 times more than the D group (Fig. 3).

So, the increase in the samples of separate groups can be written as

$\mathrm{CT}<\mathrm{D}<\mathrm{RL}=N I R L$

\section{Primary multi-laminar follicles}

The results of primary multi-laminar follicles (PFM) were similar to PF analysis (Table 1); the only difference was that the growth ratios in RL an NIRL were not equal. PFM increase in NIRL was approximately 1.46 times more than D and 1.09 times more than RL compared with CT group (Fig. 3). This result has been obtained according to Table 1, and the ascending range of the PFM can be seen in the chart. The highest value was for NIRL group. However, there was not much difference between RL and NIRL while both of them were more than D group, compared with CT (Fig. 2b).

According to the chart, the relationship between the separate groups is as

$\mathrm{CT}<\mathrm{D}<\mathrm{RL}<N I R L$
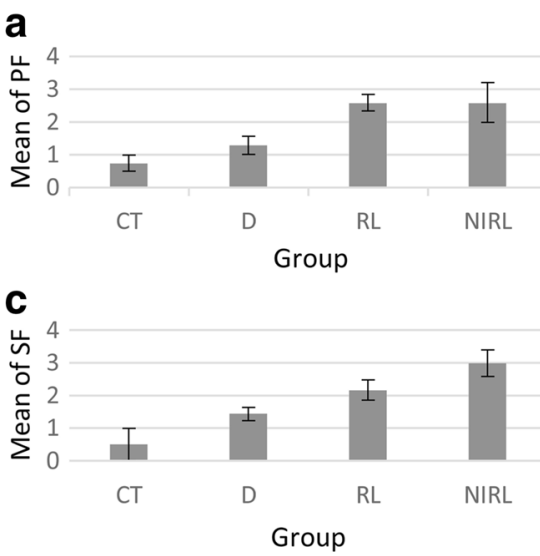

Fig. 2 Number of different follicles. a PF increase in two RL and NIRL group with equal ratio compared to D and CT group. $\mathbf{b}$ Increase ratio for PFM is not equal. There is little difference in increase ratio for NIRL and RL group compared with CT and D group apposite of PF number. $\mathbf{c}$ The
Secondary follicles

Counting secondary follicles (SF) showed that their number increased significantly in both RL and NIRL groups, compared with $\mathrm{CT}$, while there was not a significant relationship difference between RL and NIRL (Fig. 3). Compared with CT, the increasing rate in NIRL was approximately 1.38 times more than RL and 2.10 times more than D. However, there was no significant difference between D and CT or between D and RL but the difference between D and NIRL was significant (Table 1). The SF production increased almost linearly, with NIRL producing them the most (Fig. 2c).

So, the ordering of groups with respect the number of SF is as

$\mathrm{CT}<\mathrm{D}<\mathrm{RL}<N I R L$

\section{Graffian follicles}

The increase in graffian follicles (GF) differed significantly between the CT and RL and between CT and NIRL. Contrary to PF, PFM, and SF results, the difference between RL and NIRL was significant. Also, there was no significant difference between CT and D group, as in other types of follicles. Compared with CT, the increased proportion of GF in NIRL was approximately 2.50 times more than D group and 1.95 times more than RL (Fig. 3). The GF production had an ascending range, like SF and PFM. NIRL samples had maximum amounts and CT samples had minimum amounts. NIRL and RL had increased compared to D and CT. Although there was not much difference between D and RL samples (Fig. 2d), the relationship can be written as

$\mathrm{CT}<\mathrm{D}<\mathrm{RL}<N I R L$
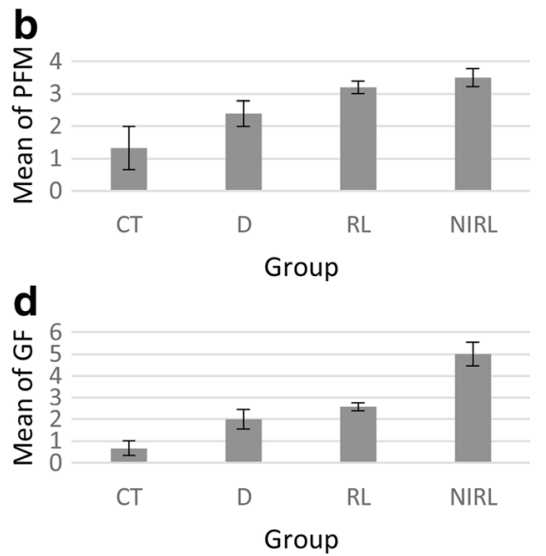

changes of SF. It increases in NIRL group more than the other group. d The changes of GF. It increases by laser and drug intervention too, but there is not little difference between D and RL group opposites of NIRL. NIRL has the most increase in the number of GF 
Table 1 Histopathological parameter in ovary tissue

\begin{tabular}{lllll}
\hline Parameters & CT & D & RL & NIRL \\
\hline PF & $0.75 \pm 0.250$ & $1.29 \pm 0.286$ & $2.60 \pm 0.245^{*}$ & $2.60 \pm 0.600^{*}$ \\
PFM & $1.33 \pm 0.667$ & $2.40 \pm 0.400$ & $3.20 \pm 0.200^{*}$ & $3.50 \pm 0.289^{*}$ \\
SF & $0.50 \pm 0.500$ & $1.43 \pm 0.202$ & $2.17 \pm 0.307^{*}$ & $3.00 \pm 0.408^{*} * *$ \\
GF & $0.67 \pm 0.333$ & $2.00 \pm 0.447$ & $2.57 \pm 0.202^{*}$ & $5.00 \pm 0.548^{*} * * * * *$ \\
\hline
\end{tabular}

Data are presented as mean $\pm \mathrm{SD}$

$C$ control, $D$ clomiphene drug, $R L$ red laser injection, $N I R L$ near-infrared laser injection

$* P<0.05$ as compared with control; $* * P<0.05$ as compared with drug group; $* * * P<0.05$ as compared with RL group

\section{Hormonal investigations}

\section{Follicle stimulation hormone}

The level of FSH production has increased in the three experimental groups compared with CT (Fig. 4a). According to the chart, growth in D samples was not much different from CT and there was a large difference between D and IRL. Compared to CT group, NIRL increased approximately 2.73 times more than RL and 25.29 times more than D. On the other hand, the increase of FSH differed significantly between $\mathrm{D}$ and RL and between $\mathrm{D}$ and IRL groups. This increase was the most for NIRL. Also, RL and the other groups differed significantly
(Table 2). According to the table, the growth rate of FSH can be written as

NIRL $>\mathrm{RL}>\mathrm{D}>\mathrm{CT}$

\section{Luteinizing hormone}

Increase in the level of luteinizing hormone (LH) differed significantly between CT and D and between CT and RL. But while LH increased in the NIRL, it was not significantly different from CT. Compared to CT group, RL increased LH approximately 1.50 times more than $\mathrm{D}$ and 6.44 times more than NIRL. Significant differences existed between D and NIRL and between RL and NIRL, while there was no significant difference between RL and D groups (Table 2). The
Fig. 3 Sections of ovary: there are four parts in this picture. a The control picture with less than different follicles. b D group. There is an increase in the growth of different follicles compared to CT. $\mathbf{c}$ RL group which shows that an increase in growth of different follicles more the D and CT group. $\mathbf{d}$ The changes of NIRL samples which have the most increase in the number of different follicles
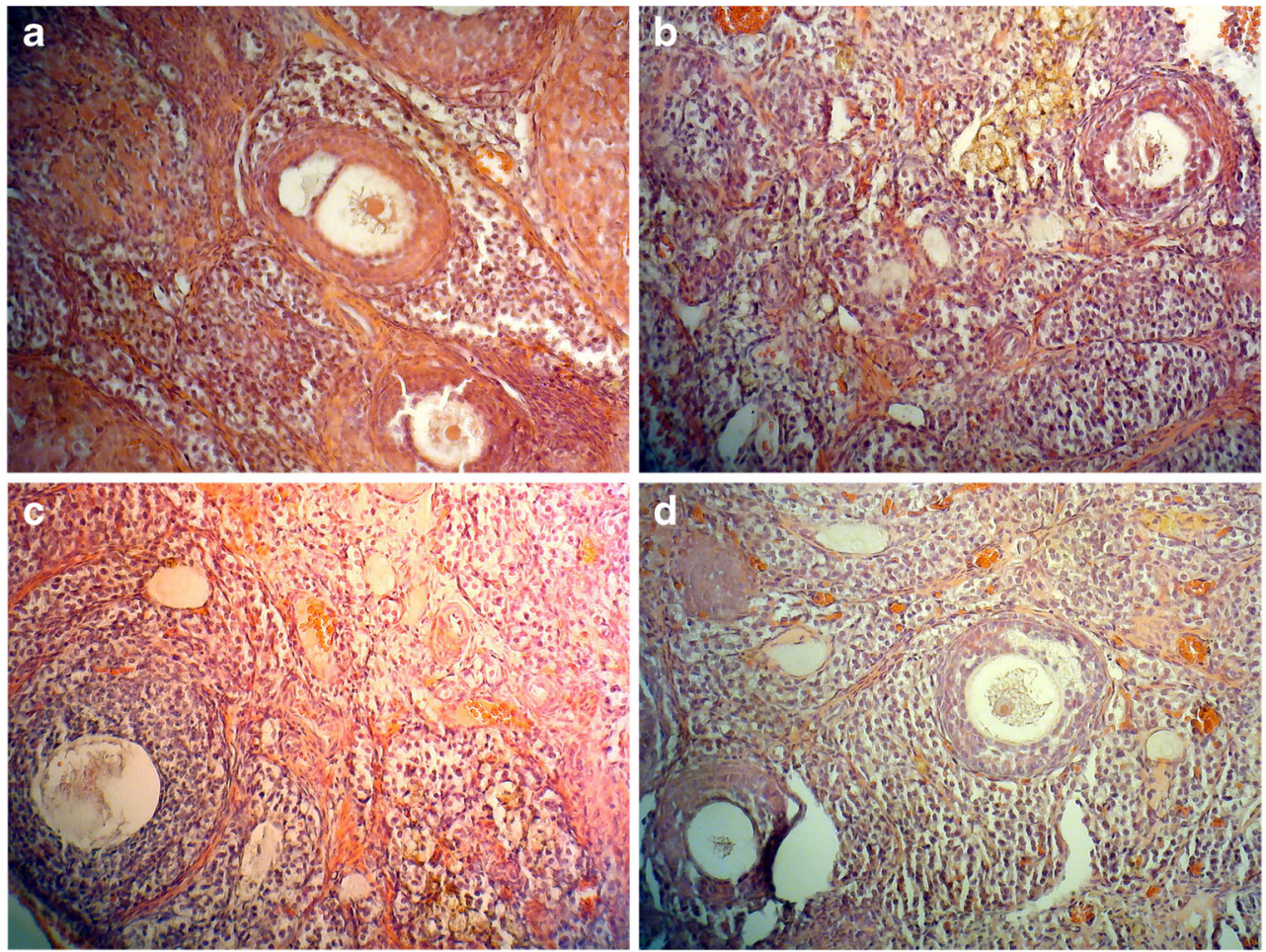
Fig. 4 Level of different hormone. a An increase in level of FSH in D, RL, and NIRL groups compared to $\mathrm{CT}$. The maximum increase is for NIRL group. b Level of LH increase by laser and drug intervention in which maximum increase is for RL group although lowest increase is in NIRL group. c An increase in level of E2 in D, RL, and NIRL groups compared to CT while there is not little difference between them. d Changes level of Pro in D, RL, and NIRL groups compared to $\mathrm{CT}$. It decreases by intervention and the most decrease is in NIRL group
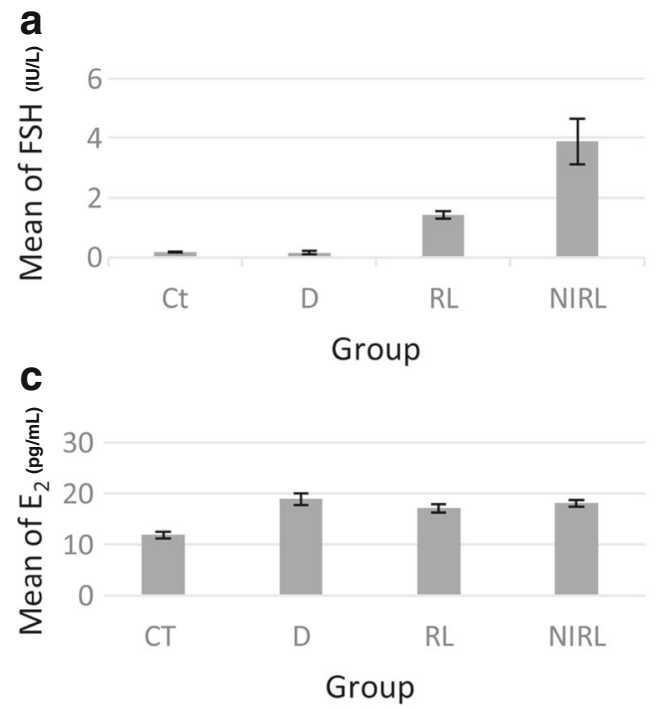

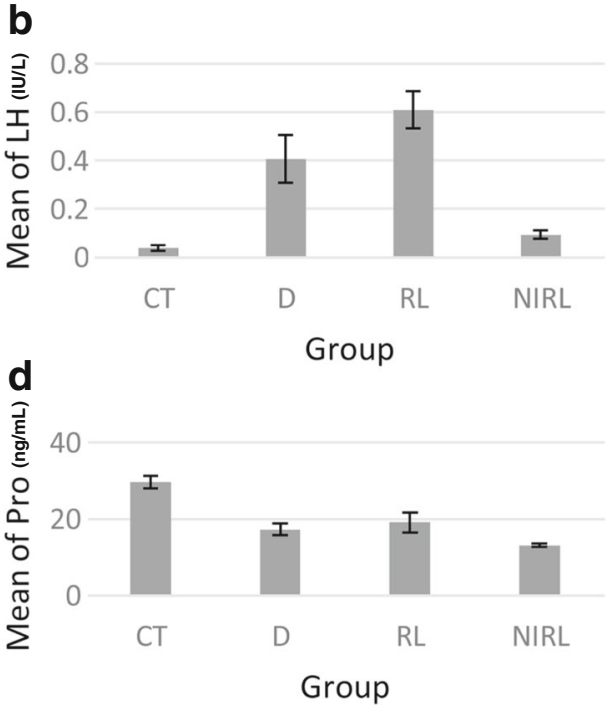

maximum $\mathrm{LH}$ level was for $\mathrm{RL}$ and the minimum was for NIRL (Fig. 4b).

The increasing rate for $\mathrm{LH}$ level was as

$\mathrm{RL}>\mathrm{D}>N I R L>\mathrm{CT}$

\section{Estrogen hormone}

Measurement of estrogen $\left(\mathrm{E}_{2}\right)$ hormone, illustrated that its increase differed significantly between CT and the other groups. Compared to CT, the maximum increase was in D samples (Fig. 4c) and the minimum was in RL and the growth rate in D was proximately 1.11 and 1.05 times more than RL and NIRL, respectively. Meanwhile, there was not much difference in the growth rate of $\mathrm{E}_{2}$ between $\mathrm{D}, \mathrm{RL}$, and NIRL groups compared with CT group (Table 2). Also, according to the result table, differences between $\mathrm{D}, \mathrm{RL}$, and NIRL groups were not significant. So, the trend for $\mathrm{E}_{2}$ increase was as

$\mathrm{D}>N I R L>\mathrm{RL}>\mathrm{CT}$

\section{Progesterone hormone}

There was significant difference between $\mathrm{CT}$ and D, between CT and RL and between CT and NIRL. NIRL group had the most decrease in progesterone (Pro) level and RL had the least. There was not much difference between RL and D samples (Fig. 4d). Ratio of decrease for NIRL was approximately 0.76 times more than $\mathrm{D}$ and 0.69 times more than RL group compared whit CT. Compared with D group, Pro decreased in NIRL and increased in RL and CT groups while there was no significant difference between $\mathrm{D}, \mathrm{RL}$ and IRL groups (Table 2). So, the decreasing rate of Pro can be written as

$N I R L>\mathrm{D}>\mathrm{RL}>\mathrm{CT}$

\section{Attenuation coefficient}

The $\ln \left(I_{0} / I\right)$ (primary and secondary intensity ratio) was plotted to $\mu x$ ( $x$ : thickness of skin). The attenuation coefficient is obtained from slope of line and presented in the chart; the

Table 2 The parameter of hormone assay in ovary tissue

\begin{tabular}{lcccc}
\hline Parameters & CT & D & RL & NIRL \\
\hline FSH (IU/L) & $0.16875 \pm 0.025174$ & $0.15329 \pm 0.052185$ & $1.42025 \pm 0.125126^{*, * *}$ & $3.87733 \pm 0.771480^{*, * * * * * * *}$ \\
LH (IU/L) & $0.03725 \pm 0.011898$ & $0.40557 \pm 0.098978^{*}$ & $0.60867 \pm 0.077197^{*}$ & $0.09457 \pm 0.018313^{* * * * * * *}$ \\
E $_{2}(\mathrm{pg} / \mathrm{mL})^{\mathrm{f}}$ & $11.900 \pm 0.7000$ & $18.917 \pm 1.1453^{*}$ & $17.120 \pm 0.8120^{*}$ & $18.086 \pm 0.6588^{*}$ \\
Pro $(\mathrm{ng} / \mathrm{mL})^{\mathrm{g}}$ & $29.6300 \pm 1.57000$ & $17.3050 \pm 1.56769^{*}$ & $19.1267 \pm 2.64739^{*}$ & $13.1760 \pm 0.41589^{*}$ \\
\hline
\end{tabular}

Data are presented as mean $\pm \mathrm{SD}$

$C$ control, $D$ clomiphene drug, $R L$ red laser intervention, $N I R L$ near-infrared laser intervention, $p g / m L 3.67 \mathrm{pmol} / \mathrm{L}, n g / m L 3.18 \mathrm{nmol} / \mathrm{L}$

$* P<0.05$ as compared with control; $* * P<0.05$ as compared with drug group; $* * * P<0.05$ as compared with RL group 
mean of measurement data is shown in Table 3. At the wavelength of $630 \mathrm{~nm}$, the attenuation coefficient was 1.1049, based on the slope of the line and 1.0879, based on the calculated data for six samples, presented in the table. Additionally, for the wavelength of $810 \mathrm{~nm}$, based on the slope of the line and data calculations for seven samples, it is 1.0299 and 1.0406, respectively (Fig. 5a, b).

\section{Conclusion}

This study investigated the effect of diode laser at two wavelengths (810 and $630 \mathrm{~nm}$ ) on folliculogenesis cycle without any surgery and compared the result with result of common drug (clomiphene) application. In the first stage, lasers had positive effect on macroscopic sexual behavior of rats. The effect of both lasers was expected to be positive, but contrary to this expectation, just near-infrared laser was significantly better than drug. It could increase different follicles in folliculogenesis cycle to produce oocyte by increasing the level of useful sex hormones. Rats' responses to nearinfrared laser (NIR group) in producing different follicles at the end of folliculogenesis cycle were approximately $57 \%$ positive, $29 \%$ neutral, and $14 \%$ negative. With red laser (RL group) responses were $43 \%$ positive, $14 \%$ neutral, and $43 \%$ negative. By drug intervention, they were 43 positive, $29 \%$ neutral, and $14 \%$ negative. Although, the results varied for the two laser modes, the method and statistical analyses that were used in this study showed significant increasing results for NIRL that certainly can be used in future studies for finding a cure to ovarian negligence to produce more oocyte and treat diseases caused by it like PCOS. Hence, this can be investigated on rats with PCOS disease to find certain cure for that.

Compared with previous studies, like someone $[9,17,20$, $21,23]$ in which laser was used as a complementary treatment, the present study showed that diode laser with $810 \mathrm{~nm}$ wavelength increases the function of folliculogenesis cycle to produce oocyte significantly more than drug.

In interpreting the findings of this study, it is important to take its limitations into consideration. For instance, the exact optical receptor and their optical potential on ovary tissue to produce oocyte are not known. Also, ovary cannot be exactly located due to its small size and its motion on mm range in the body of rat. Additionally, while the result of study was significant and acceptable, the limited sample size bars definitive conclusion. Furthermore, rats were kept in natural environment in the laboratory. However, many potentially important exposure routes were not evaluated. Such exposures include information about dietary intake, blood pressure, and the organs under the skin of irradiated rats, as exposure may vary due to different amounts of tissues such as fat.

The number of ovarian variable follicles in our study exhibited different effects of treatments examined in this research. Given that it is unknown if the ovaries were sick, results are not definite; but based on previous studies [9, 20, 34 ], our results can be useful in the treatment of hypo activation of ovary.

Finally, this study investigated the direct effect of lasers in near-infrared and red spectrum on producing different follicles in folliculogenesis cycle and did not consider disorders in

Table 3 Attenuation coefficient

\begin{tabular}{llllll}
\hline Wavelength $(\mathrm{nm})$ & $I_{0}\left(\mathrm{w} / \mathrm{cm}^{2}\right)$ & $I\left(\mathrm{w} / \mathrm{cm}^{2}\right)$ & $\ln I_{0} / I$ & $x \pm 0.03(\mathrm{~mm})$ & $\mu(1 / \mathrm{mm})$ \\
\hline 630 & 32.3 & 7.8 & 1.4209 & 1.26 & 1.12770 \\
& 32.7 & 11.2 & 1.0715 & 1.1 & 0.97410 \\
& 31.6 & 8.7 & 1.2898 & 1.19 & 1.08387 \\
& 34.2 & 8.3 & 1.416 & 1.28 & 1.10625 \\
& 33.5 & 5.3 & 1.8438 & 1.51 & 1.22106 \\
& 32.1 & 10.2 & 1.1465 & 1.13 & 1.01460 \\
& & & Mean of $\mu=1.088 \pm 0.152$ \\
& 202 & 50 & 1.3962 & 1.36 & 1.026618 \\
& 216 & 67 & 1.1706 & 1.1 & 1.064182 \\
& 188 & 61 & 1.1256 & 1.1 & 1.023273 \\
& 216 & 55 & 1.3679 & 1.28 & 1.068672 \\
& 219 & 67 & 1.1844 & 1.17 & 1.012308 \\
& 195 & 64 & 1.1141 & 1.04 & 1.07125 \\
& 218 & & 1.1504 & 1.13 & Mean of $\mu=1.037 \pm 0.138$
\end{tabular}

Measurement of six samples for red laser by $630 \mathrm{~nm}$ wavelength and seven samples for near-infrared laser by $810 \mathrm{~nm}$ wavelength 
Fig. 5 Attenuation coefficient. a The attenuation coefficient for red laser by $630 \mathrm{~nm}$ wavelength. b The attenuation coefficient for near-infrared laser by $810 \mathrm{~nm}$ wavelength

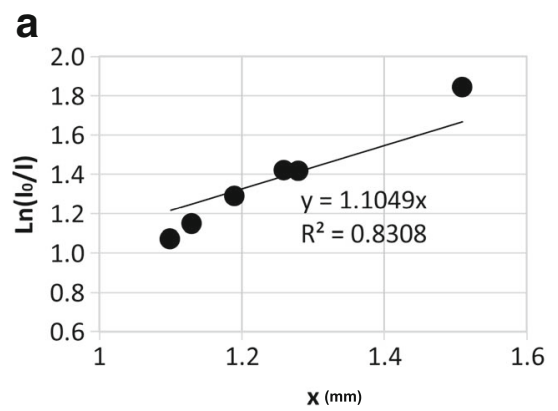

b

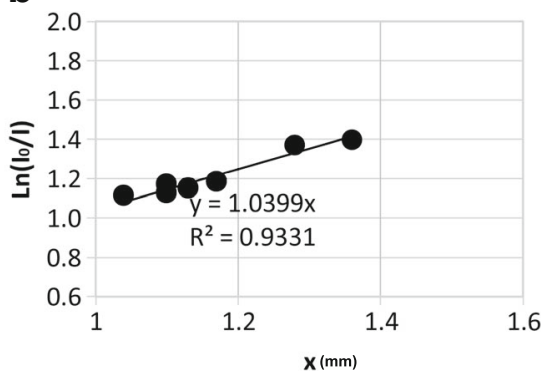

folliculogenesis cycle to produce oocyte which can later be investigated.

Despite these limitations, this study is an important contribution to previous methods in the treatment of diseases caused by negligence of the ovary. One of these diseases is polycystic ovarian syndrome (PCOS) for which laser had been used as complementary therapy $[20,34]$. The aim of this research was to propose the use of lasers autonomously, without any medical and surgical intervention, in the treatment of ovarian negligence in adult women, unlike the past studies [9, 20].

Acknowledgments This study was supported by a grant from Tabriz University of Medical Sciences, grant code: 5/89/137. Hereby the authors express their gratitude to Eshrat Khah and Musavi, Doctoral candidates, at the University of Tabriz for measuring hormone level; Abed Ellahi of the anatomy group, Tabriz University, and Pedram Hamidi, master candidate at the Shahrood University, for designing rat restrainer and processing results by the SPSS software.

\section{Compliance with ethical standards}

Conflict of interest The authors declare that they have no conflict of interest.

Ethical approval The research protocol of this study was approved by Ethics in Research Committee of Tabriz University of Medical Sciences TUMS, under code number: TBZMED.REC.1394.238.

Open Access This article is distributed under the terms of the Creative Commons Attribution 4.0 International License (http:// creativecommons.org/licenses/by/4.0/), which permits unrestricted use, distribution, and reproduction in any medium, provided you give appropriate credit to the original author(s) and the source, provide a link to the Creative Commons license, and indicate if changes were made.

\section{References}

1. Erickson GF and Shimasaki S (2000) The role of the oocyte in folliculogenesis. Trends Endocrinol Metab 11:193-198. doi:10. 1016/S1043-2760(00)00249-6

2. Te Velde E, Scheffer G, Dorland M et al (1998) Developmental and endocrine aspects of normal ovarian aging. Mol Cell Endocrinol 145:67-73. doi:10.1016/S0303-7207(98)00171-3

3. Heeren AM, Van Iperen L, Klootwijk DB et al (2015) Development of the follicular basement membrane during human gametogenesis and early folliculogenesis. BMC Dev Biol 15:4. doi:10.1186/ s12861-015-0054-0

4. Byskov AG (1986) Differentiation of mammalian embryonic gonad. Physiol Rev 66:71-117

5. Bonnet A, Servin B, Mulsant P et al (2015) Spatio-temporal gene expression profiling during in vivo early ovarian folliculogenesis: integrated transcriptomic study and molecular signature of early follicular growth. PloS one 10:e141482. doi:10.1371/journal. pone. 0141482

6. Choi Y and Rajkovic A (2006) Genetics of early mammalian folliculogenesis. Cell Mol Life Sci 63:579-590. doi:10.1007/ s00018-005-5394-7

7. Li H, Moll J, Winkler A et al (2015) RHAMM deficiency disrupts folliculogenesis resulting in female hypofertility. Biol Open. doi:10. 1242/bio.201410892

8. Sánchez F, Romero S, De Vos M et al (2015) Human cumulusenclosed germinal vesicle oocytes from early antral follicles reveal heterogeneous cellular and molecular features associated with in vitro maturation capacity. Hum Reprod 30:1396-1409

9. Sherein S (2009) Comparative studies on the therapeutic effect of low power laser biostimulation and clomid on the treatment of polycystic ovarian disease. Egypt J Comp Pathol Clin Pathol 22(1):113-129

10. Broekmans F, Knauff E, Valkenburg O et al (2006) PCOS according to the Rotterdam consensus criteria: change in prevalence among WHO-II anovulation and association with metabolic factors. BJOG Int J Obstet Gynaecol 113:1210-1217. doi:10.1111/j.14710528.2006.01008.x

11. Kawamura K, Cheng Y, Suzuki N et al (2013) Hippo signaling disruption and Akt stimulation of ovarian follicles for infertility treatment. Proc Natl Acad Sci 110:17474-17479. doi:10.1073/ pnas. 1312830110

12. Brosens I and Benagiano G (2015) Menstrual preconditioning for the prevention of major obstetrical syndromes in polycystic ovary syndrome. Am J Obstet Gynecol 213:488-493. doi:10.1016/j.ajog. 2015.07.021

13. Farquhar C, Lilford R, Marjoribanks J et al (2007) Laparoscopic 'drilling' by diathermy or laser for ovulation induction in anovulatory polycystic ovary syndrome. Cochrane Database Syst Rev 3: CD001122

14. Clayton W, Lipton M, Elford J et al (2005) A randomized controlled trial of laser treatment among hirsute women with polycystic ovary syndrome. Br J Dermatol 152:986-992

15. Fukaya T, Murakami T, Tamura M et al (1995) Laser vaporization of the ovarian surface in polycystic ovary disease results in reduced ovarian hyperstimulation and improved pregnancy rates. Am J Obstet Gynecol 173:119-125. doi:10.1016/0002-9378(95)90179-5

16. Hariri LP, Liebmann ER, Marion SL et al (2010) Simultaneous optical coherence tomography and laser induced fluorescence imaging in rat model of ovarian carcinogenesis. Cancer Biol Ther 10: 438-447. doi:10.4161/cbt.10.5.12531 
17. Qiu F, Liu H-Y, Dong Z-N et al (2009) Searching for potential ovarian cancer biomarkers with matrix-assisted laser desorption/ ionization time-of-flight mass spectrometry. Am J Biomed Sci 1: 80. doi:10.5099/aj090100080

18. Keckstein G, Rossmanith W, Spatzier K et al (1990) The effect of laparoscopic treatment of polycystic ovarian disease by CO 2-laser or Nd: YAG laser. Surg Endosc 4:103-107

19. Zhu W, Fu Z, Chen X et al (2010) Transvaginal ultrasound-guided ovarian interstitial laser treatment in anovulatory women with polycystic ovary syndrome: a randomized clinical trial on the effect of laser dose used on the outcome. Fertil Steril 94:268-275

20. Zhu W, Li X, Chen X et al (2006) Ovarian interstitial YAG-laser: an effective new method to manage anovulation in women with polycystic ovary syndrome. Am J Obstet Gynecol 195:458-463. doi:10. 1016/j.ajog.2006.01.022

21. Liebert AD, Bicknell BT and Adams RD (2014) Protein conformational modulation by photons: A mechanism for laser treatment effects. Med Hypotheses 82:275-281. doi:10.1016/j.mehy.2013.12.009

22. Loaiza LA, Yamaguchi S, Ito M et al (2002) Electro-acupuncture stimulation to muscle afferents in anesthetized rats modulates the blood flow to the knee joint through autonomic reflexes and nitric oxide. Auton Neurosci 97:103-109. doi:10.1016/S1566-0702(02) 00051-6

23. Shen C-C, Yang Y-C, Chiao M-T et al (2013) Low-level laser stimulation on adipose-tissue-derived stem cell treatments for focal cerebral ischemia in rats. Evid Based Complement Alternat Med 2013. doi:10.1155/2013/594906

24. Stener-Victorin E, Kobayashi R and Kurosawa M (2003) Ovarian blood flow responses to electro-acupuncture stimulation at different frequencies and intensities in anaesthetized rats. Auton Neurosci 108:50-56. doi:10.1016/j.autneu.2003.08.006

25. Yang MY and Rajamahendran R (2000) Morphological and Biochemical Identification of Apoptosis in Small, Medium, and
Large Bovine Follicles and the Effects of Follicle-Stimulating Hormone and Insulin-Like Growth Factor-I on Spontaneous Apoptosis in Cultured Bovine Granulosa Cells 1. Biol Reprod 62: 1209-1217. doi:10.1095/biolreprod62.5.1209

26. Moon Y, Duleba A, Yun Y et al (1989) Effects of clomiphene citrate on ovarian function in hypophysectomized rats. J Reprod Fertil 86: 753-757

27. Salehpour F, Rasta SH, Mohaddes G et al (2016) Therapeutic effects of $10-\mathrm{Hz}$ Pulsed wave lasers in rat depression model: A comparison between near-infrared and red wavelengths. Lasers Surg Med 48:695-705. doi:10.1002/lsm.22542

28. Kolibianakis EM, Papanikolaou EG, Fatemi HM et al (2005) Estrogen and folliculogenesis: is one necessary for the other? Curr Opin Obstet Gynecol 17:249-253

29. Palter SF, Tavares AB, Hourvitz A et al (2001) Are estrogens of import to primate/human ovarian folliculogenesis? Endocr Rev 22: 389-424. doi:10.1210/edrv.22.3.0433

30. Filicori M (1999) The role of luteinizing hormone in folliculogenesis and ovulation induction. Fertil Steril 71:405-414

31. Koering MJ, Danforth DR and Hodgen GD (1991) Early folliculogenesis in primate ovaries: testing the role of estrogen. Biol Reprod 45:890-897

32. Dizerega GS and Hodgen GD (1982) The interovarian progesterone gradient: a spatial and temporal regulator of folliculogenesis in the primate ovarian cycle. J Clin Endocrinol 54:495-499

33. Hillier S (1994) Current concepts of the roles of follicle stimulating hormone and luteinizing hormone in folliculogenesis. Hum Reprod 9:188-191. doi:10.1093/oxfordjournals.humrep.a138480

34. Farquhar C, Lilford R, Marjoribanks J et al (2007) Laparoscopic drilling by diathermy or laser for ovulation induction in anovulatory polycystic ovary syndrome. Cochrane Libr. doi:10.1002/14651858. CD001122.pub3 